\title{
Unilateral blaschkoid lichen planus
}

\section{Katarzyna Borowska', Piotr Brzeziński²}

${ }^{1}$ Department of Histology and Embryology with Experimental Cytology Unit, Medical University of Lublin, Lublin, Poland, ${ }^{2}$ Department of Physiotherapy and Medical Emergency, Faculty of Health Sciences, Pomeranian Academy, Slupsk, Poland

Corresponding author: Piotr Brzeziński, MD PhD, E-mail: brzezoo77@yahoo.com

\begin{abstract}
Lichen planus (LP) is a chronic, inflammatory, autoimmune disease that affects the skin, oral and/or genital mucosa, scalp, and nails. The linear variety of LP can simply be a result of Koebner's phenomenon, segmental LP or zosteriform LP. We present a case of a 55-year-old patient with unilateral blaschkoid lichen planus. LP in a Blaschkoid distribution is rare with only some case reports published till date.
\end{abstract}

Key words: Unilateral blaschkoid lichen planus, Inflammation

\section{INTRODUCTION}

Lichen planus (LP) is a chronic, inflammatory, autoimmune disease that affects the skin, oral and/ or genital mucosa, scalp, and nails. Histologically, the disease is characterized by a inflammatory infiltrate and vacuolar degeneration of the basal layer of the epidermis. The diagnosis is based on the clinical presentation and characteristic histological findings. LP preferentially affects middleaged adults, with no known gender predisposition [1-5]. The current first line treatment are topical and/or systemic corticosteroids.

\section{CASE REPORT}

A 55-year-old patient presented with multiple redpurple colored lesions on the right side of his trunk which he had noticed about one month back. These lesions were associated with itching. He had no infectious symptoms, such as throat pain and fever, prior to the development of the dermatosis. Physical examination revealed that a number of reddish papules and erythematous lesions with slight scales were distributed along Blaschko's lines (Figs. la and lb). The scalp, hair, mucosae and nails were normal. Routine laboratory investigations were unremarkable. Serology for HIV, Hepatitis B and C was negative.

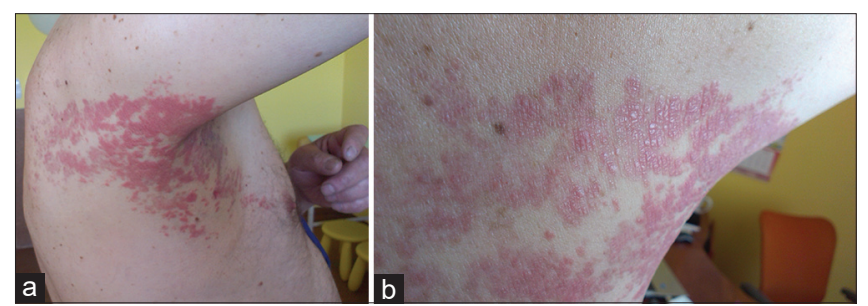

Figure 1: (a-b). Violaceous papules and plaques lesions restricting only to right half of the body.

Other dermatitis showing linear distribution, such as inflammatory linear verrucous epidermal nevus, linear lichen striatus, and linear lichen planus were all ruled out. Based on the clinical and histopathological findings, a diagnosis of Blaschkoid LP. The patient was successfully treated with topical corticosteroid (betamethasone butyrate propionate) ointment and oral antihistamines. The patient responded favorably to treatment.

\section{DISCUSSION}

Some congenital nevi and inflammatory diseases like psoriasis, vitiligo and LP may present in a nevoid form [1]. The linear variety of LP can simply be a result of Koebner's phenomenon, segmental LP or zosteriform LP. It must also be differentiated with lichenoid 
epidermal nevus, lichen striatus, linear psoriasis or inflammatory linear verrucous epidermal

nevus $[1,2]$. The many cases of lichen planus along Blaschko lines are misdiagnosed as zosteriform lichen planus. Lichen planus in a Blaschkoid distribution is rare with only some case reports published till date [1-3]. This case was deemed worthy of presentation because of the rarity of the condition.

\section{Consent}

The examination of the patient was conducted according to the principles of the Declaration of Helsinki.

The authors certify that they have obtained all appropriate patient consent forms, in which the patients gave their consent for images and other clinical information to be included in the journal. The patients understand that their names and initials will not be published and due effort will be made to conceal their identity, but that anonymity cannot be guaranteed.

\section{REFERENCES}

1. Belmourida S, Meziane M, Benzekri L, Ismaili N, Senouci K. Linear lichen planus. Our Dermatol Online. 2021;12:e25.

2. Adil M, Amin SS, Arif T. Blaschkoid lichen planus: A rare presentation of a common disease. Our Dermatol Online. 2016;7:349-50.

3. Kumavat S. Unilateral blaschkoid lichen planus. Indian Dermatol Online J. 2019;10:606-7.

4. Boyd AS, Neldner KH. Lichen planus. J Am Acad Dermatol. 1991;25:593-619.

5. Ioannides D, Vakirlis E, Kemeny L, Marinovic B, Massone C, Murphy R, et al. European S1 guidelines on the management of lichen planus: a cooperation of the European Dermatology Forum with the European Academy of Dermatology and Venereology. J Eur Acad Dermatol Venereol. 2020;34:1403-14.

Copyright by Katarzyna Borowska, et al. This is an open-access article distributed under the terms of the Creative Commons Attribution License, which permits unrestricted use, distribution, and reproduction in any medium, provided the original author and source are credited.

Source of Support: Nil, Conflict of Interest: None declared. 